\title{
Relationships between changes in guest ion properties and in host framework topology in ionic coordination polymers
}

\section{Supporting Information}

\author{
Pavel N. Zolotarev
}

Samara Center for Theoretical Materials Science, Samara State Technical University, Molodogvardeyskaya Str. 244, 443100 Samara, Russia 
Fig. S1. (a) Distribution of the frameworks depending on the number of unique ions per framework. (b) Distribution of the frameworks depending on the number of unique ions per framework with a given topology.

a)

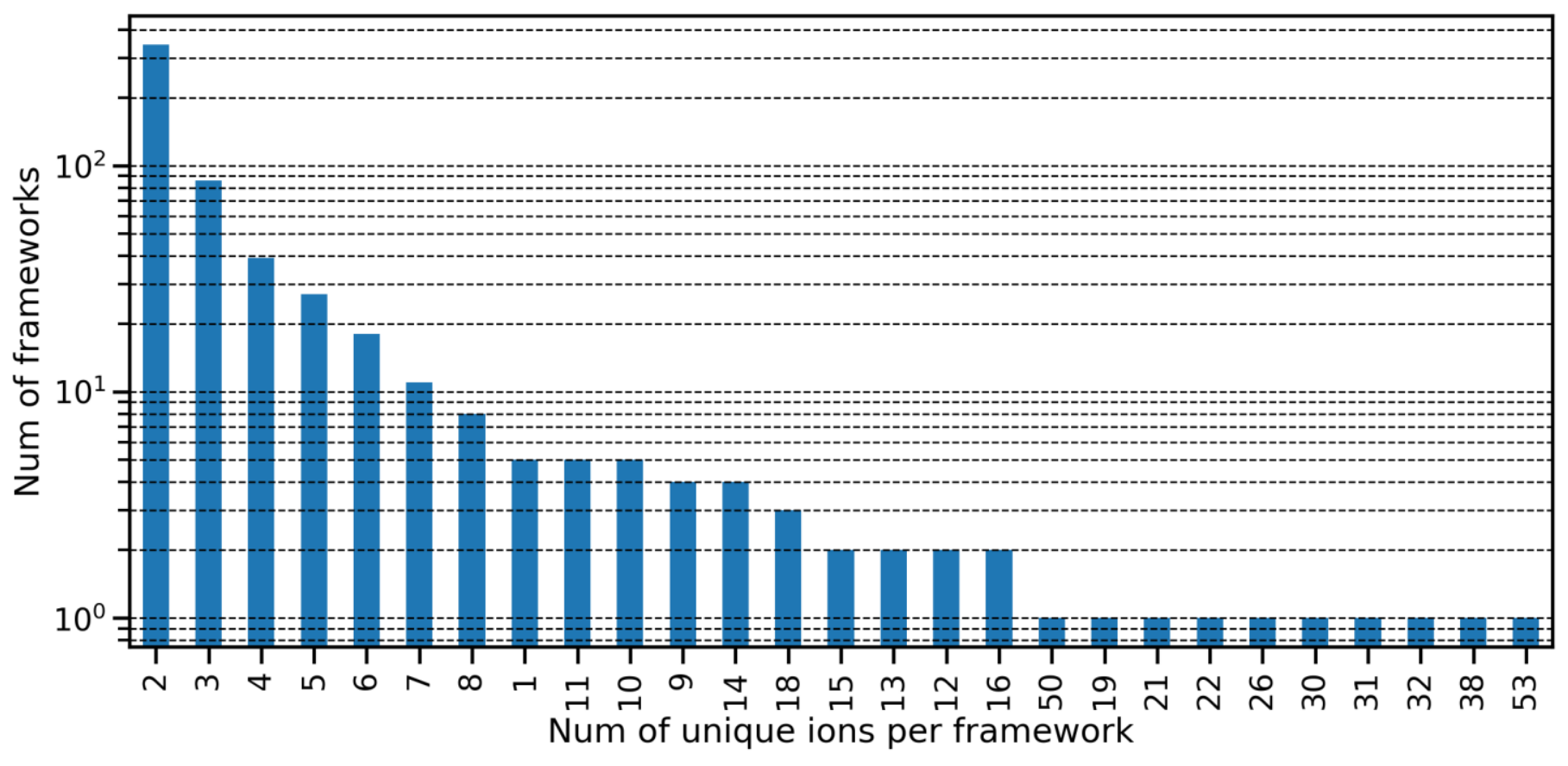

b)

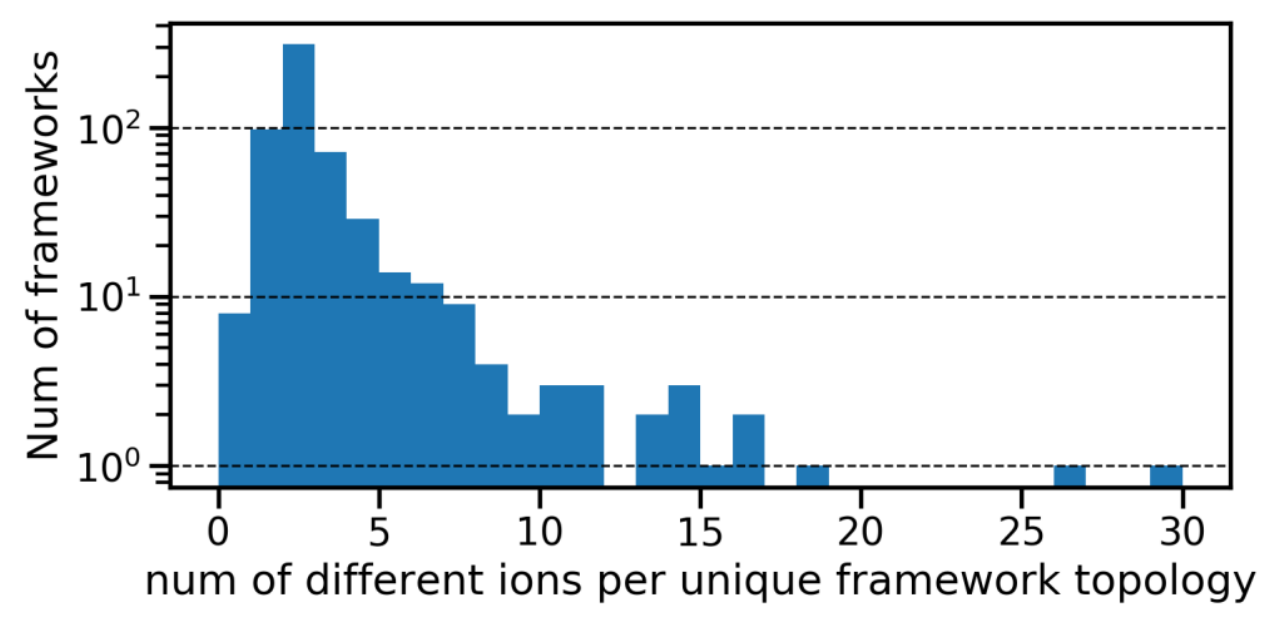


Fig. S2. Pairwise Spearman's rank correlation coefficients $(\rho)$ calculated for initial set of descriptors. The correlation coefficients are shown only for descriptor pairs with $|\rho|>0.8$.

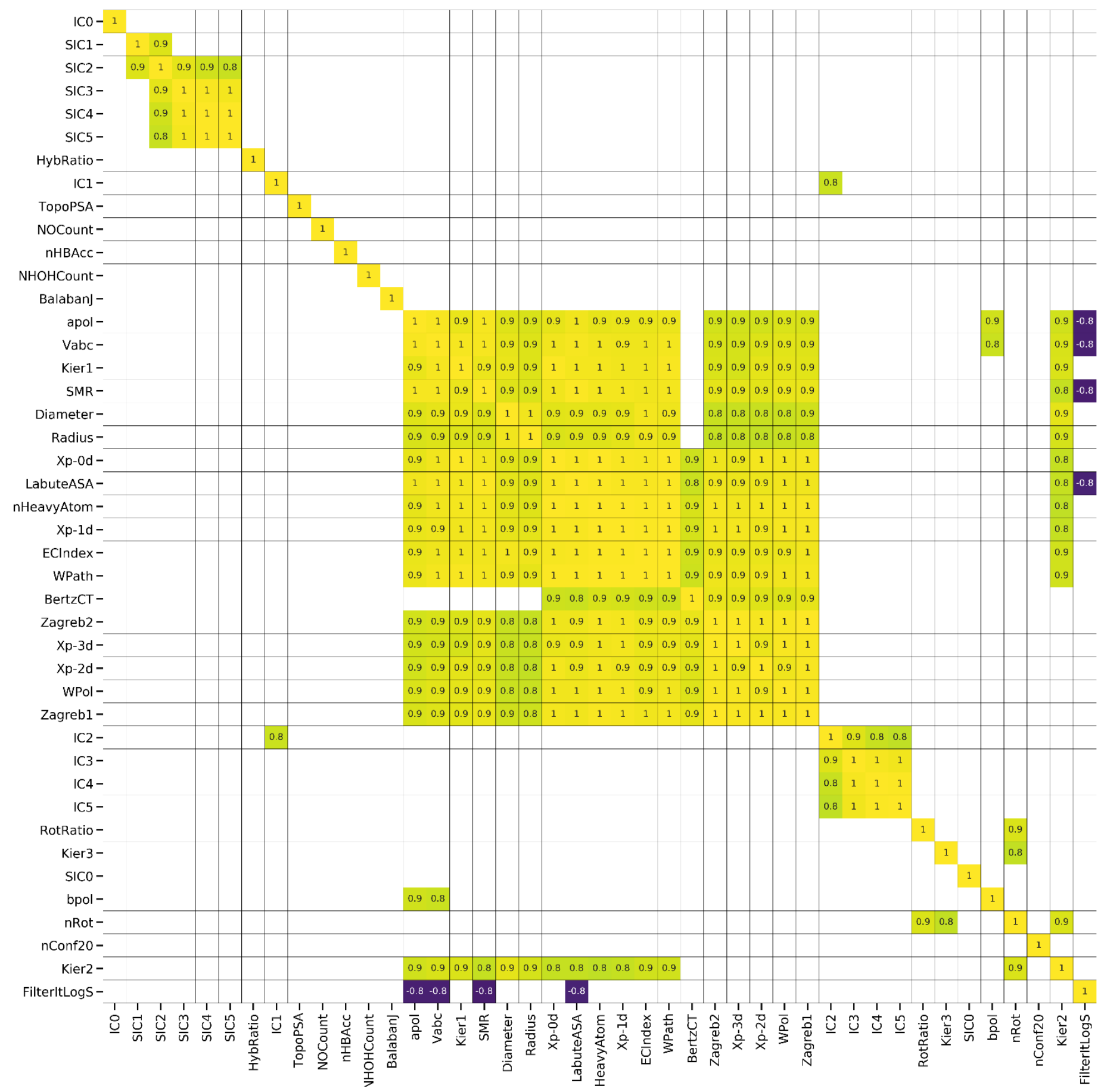


Fig. S3. The validation of the trained ML models using y-randomization to assess the significance of the classifiers' performance. The performance of a trained model is evaluated on a test set using the $\mathrm{F}_{1}$ score. The $80 / 20$ train/test split is performed at each of the 50 repeats of shuffling training set $y$-labels. We can see that the model trained on the unperturbed data (red line) is able to capture some relationships and significantly outperform the models trained on data with permuted target labels (blue bars).

$\Delta$ dim prediction
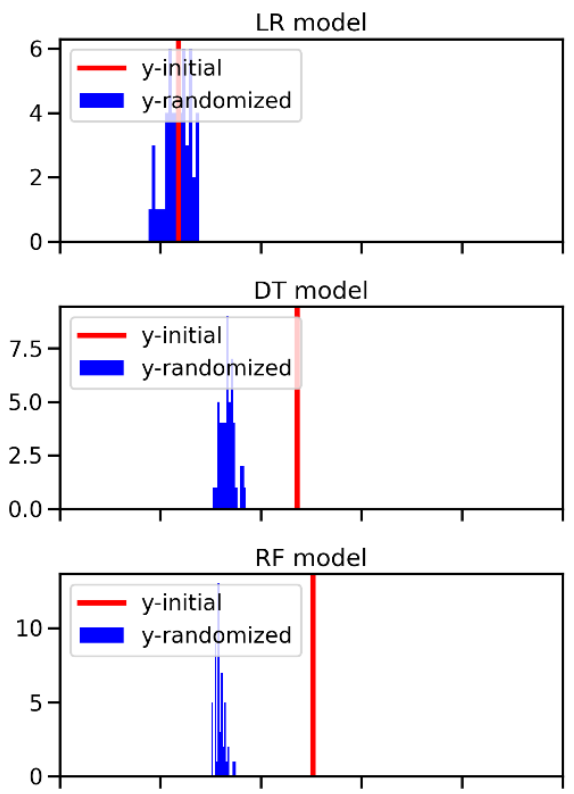

SVC model
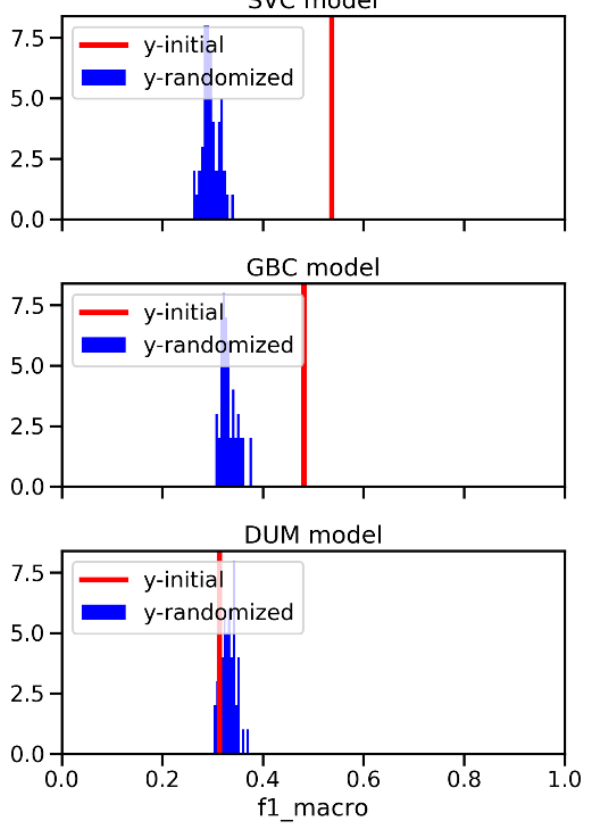

$\Delta$ TD10 prediction
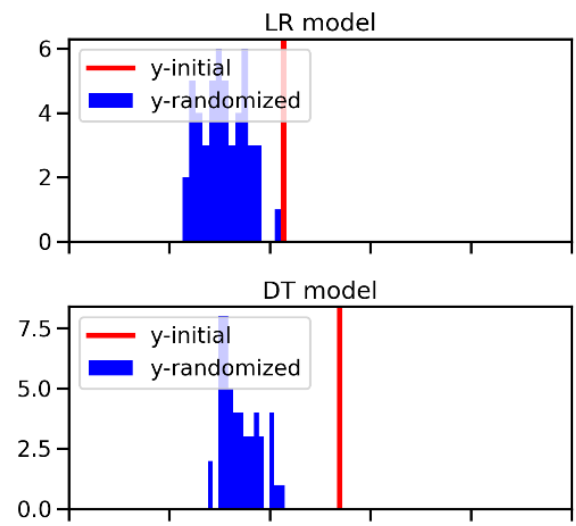

RF model

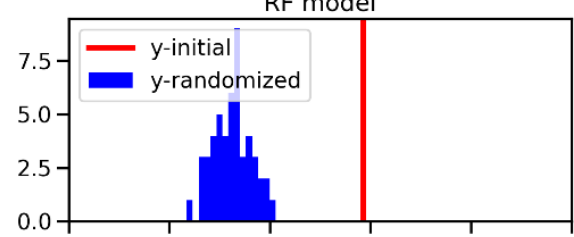

SVC model
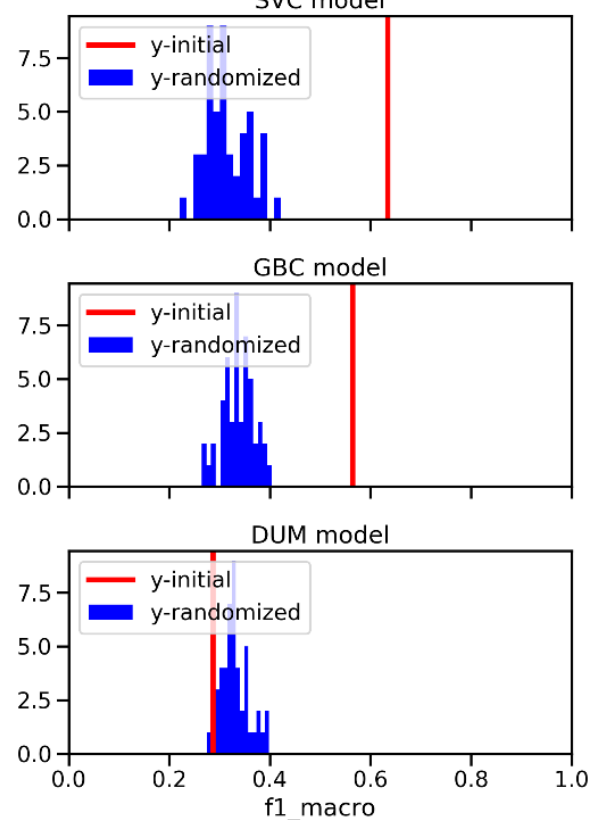

$\Delta$ ring size prediction
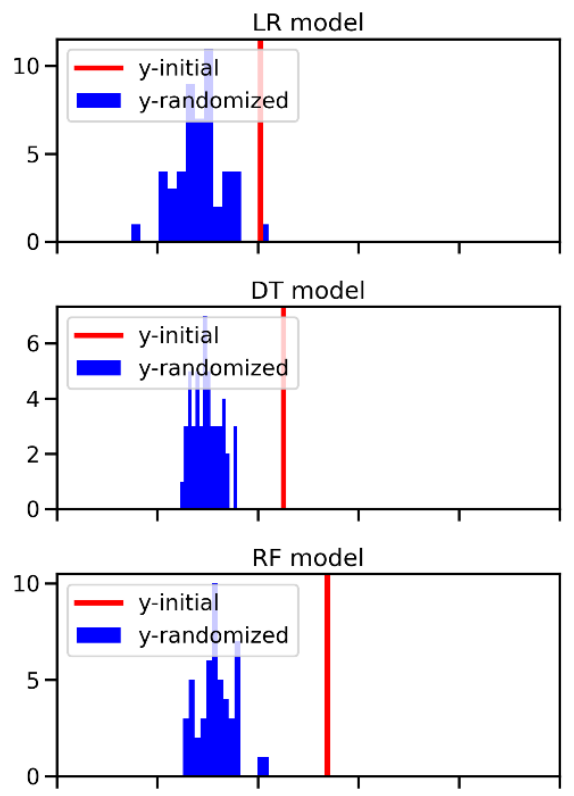

SVC model
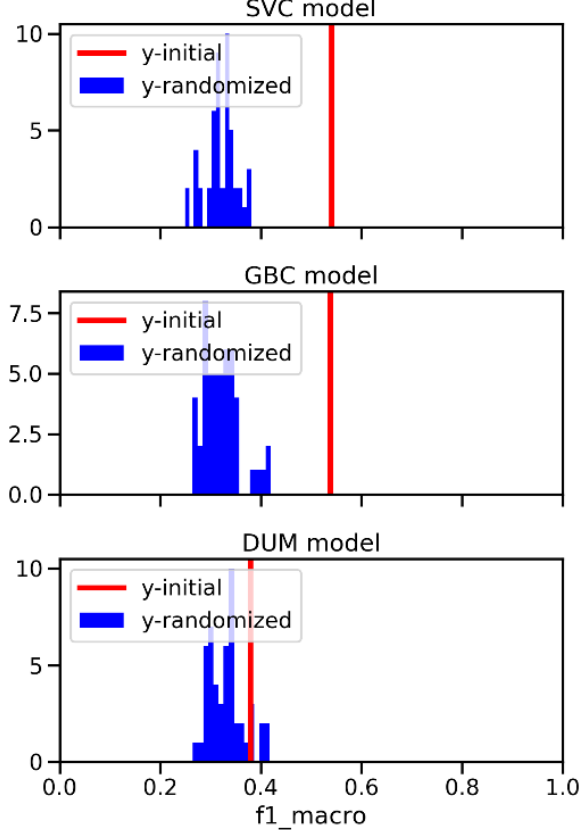
Fig. S4. Macro-averaged $F_{1}$ score values with error bars ( 1 standard deviation) calculated by means of the $5 \times 5$ nested cross-validation for the selected set of classifiers. Note, that the classifiers were trained on a feature matrix filled with random numbers.

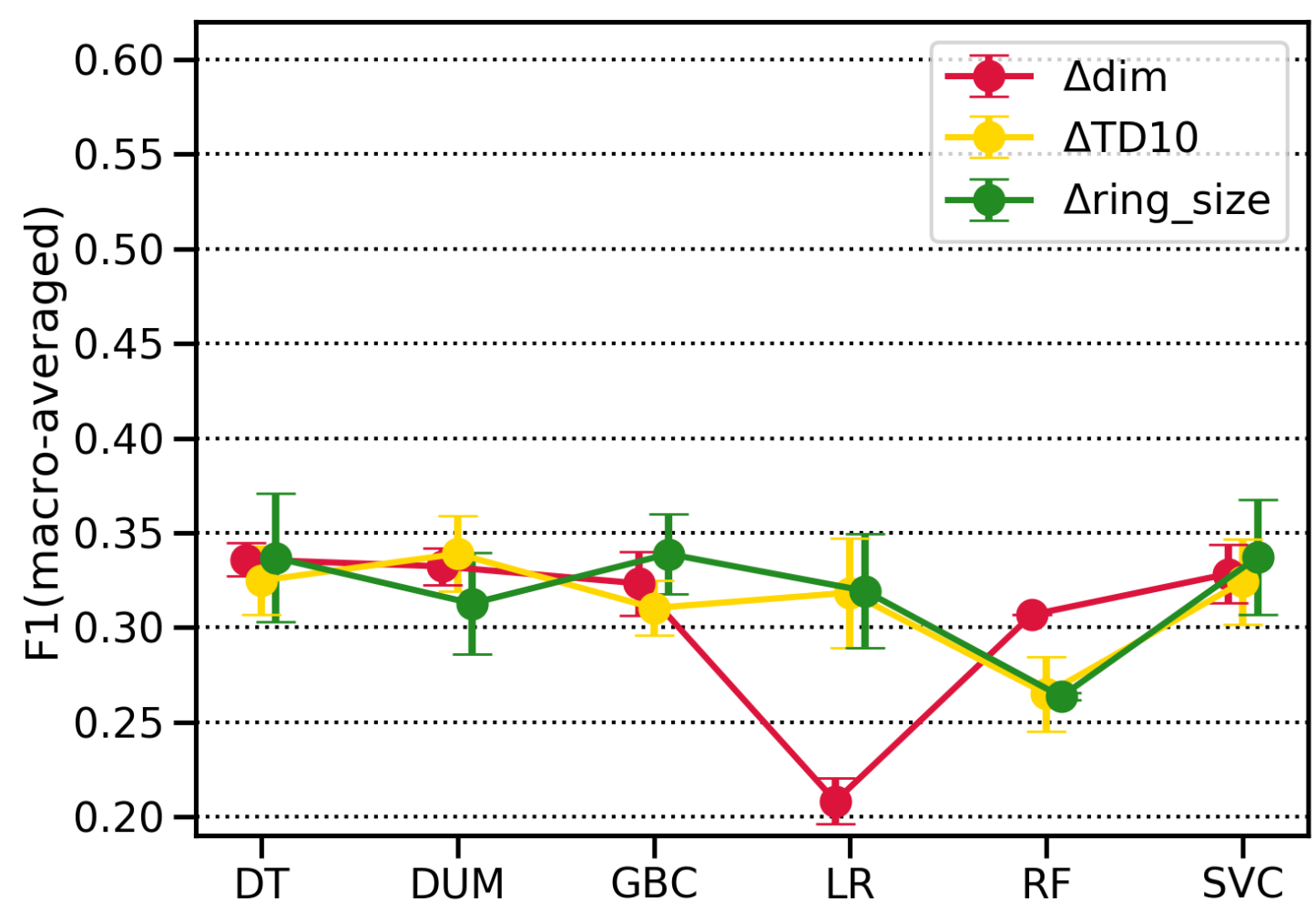


Fig. S5. Learning curves built for the trained classifiers predicting changes in dim, TD10, and ring_size framework properties.
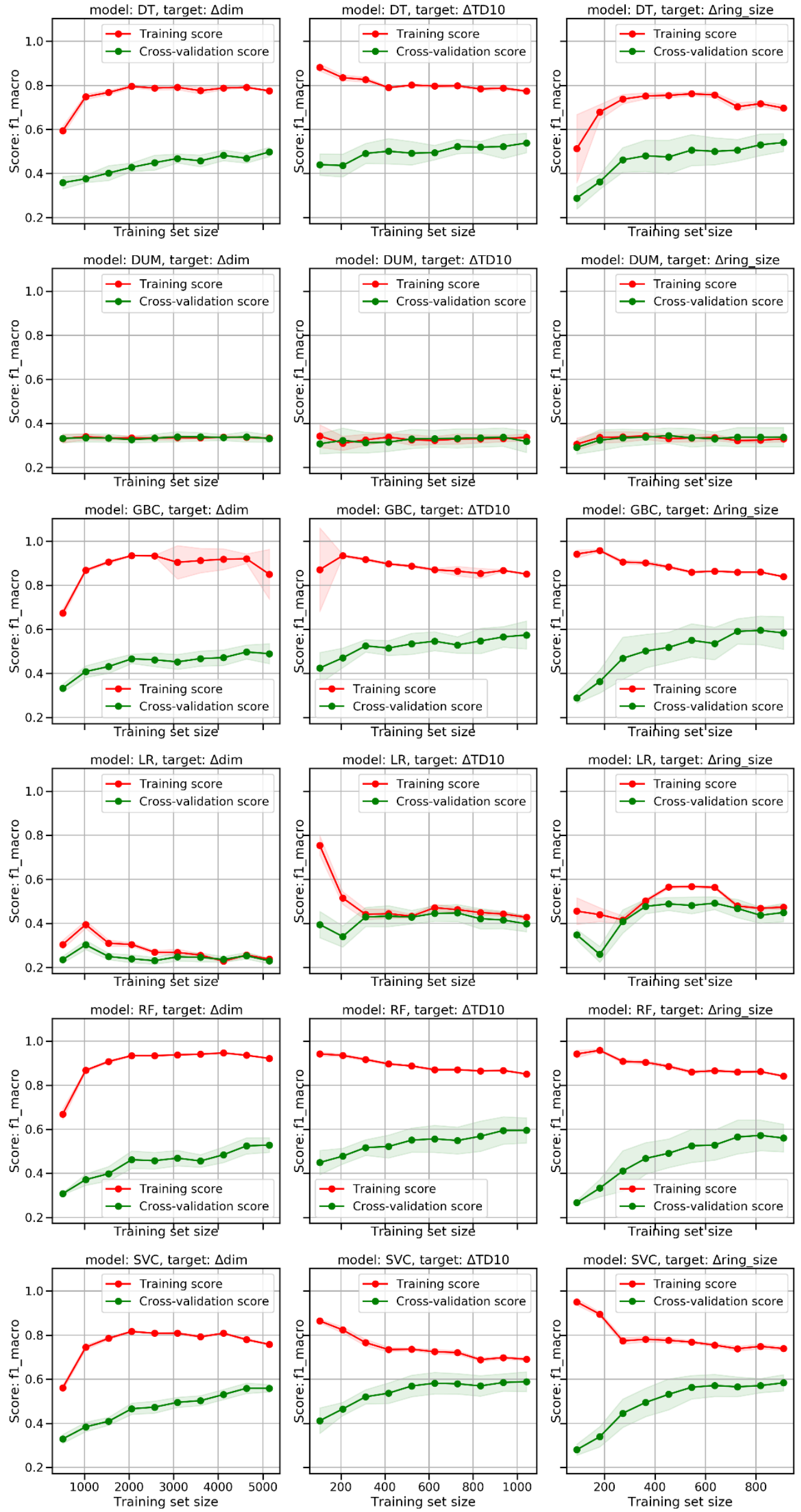
Fig. S6. Permutation feature importances for each classifier used in prediction of (a) $\Delta$ dim, (b) $\Delta$ TD10, and (c) $\Delta$ ring_size framework properties.

a)
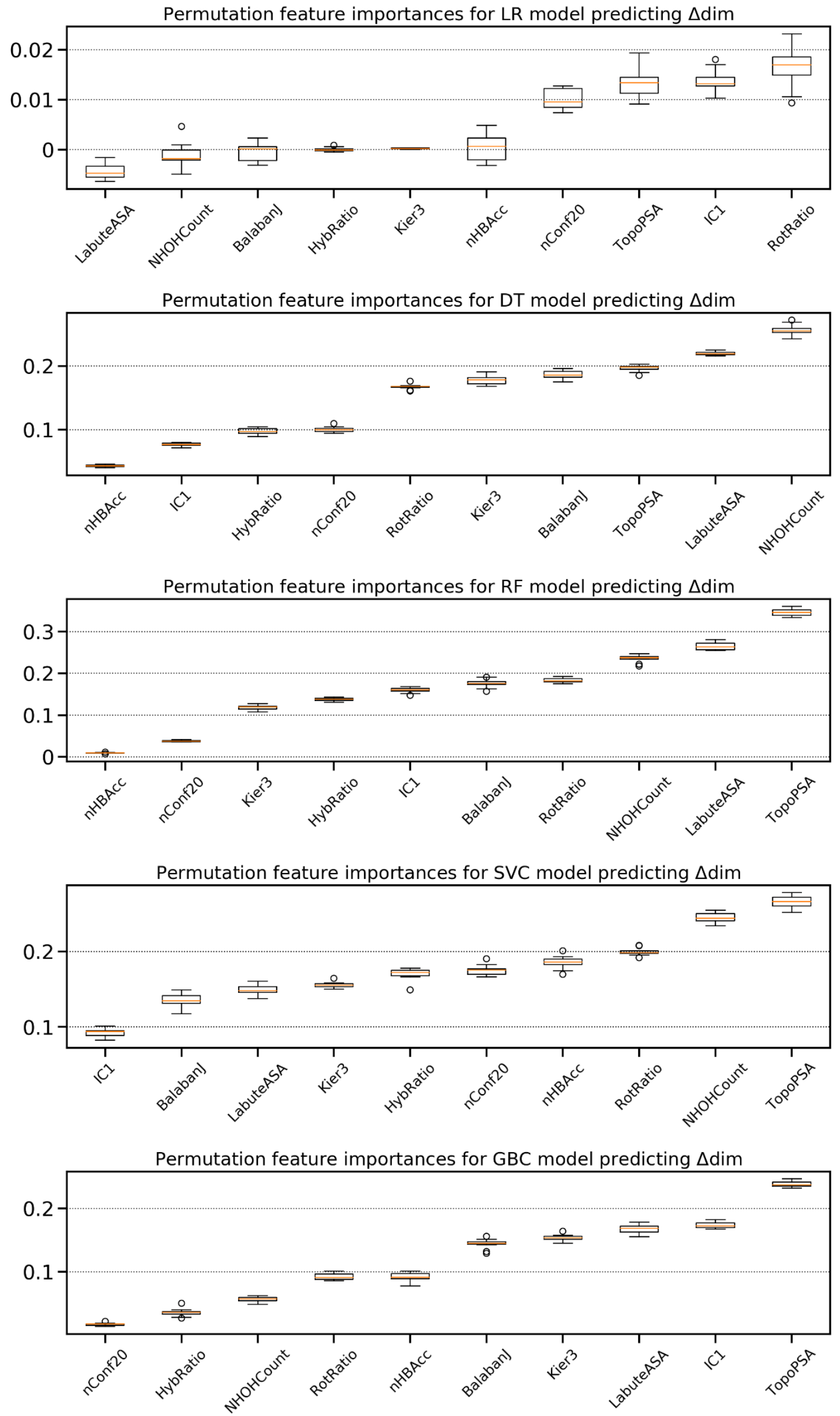
b)
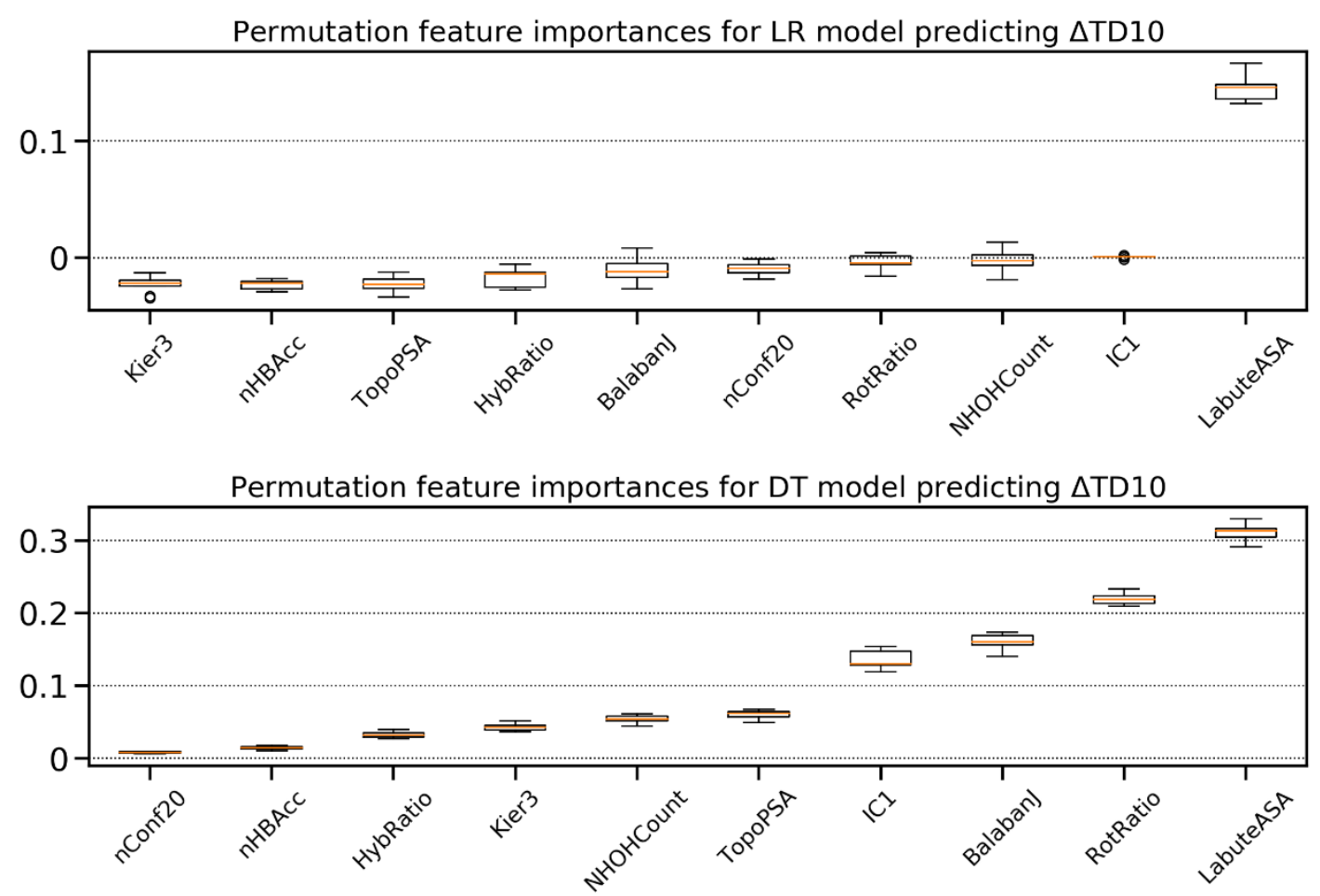

Permutation feature importances for RF model predicting $\triangle T$ TD10

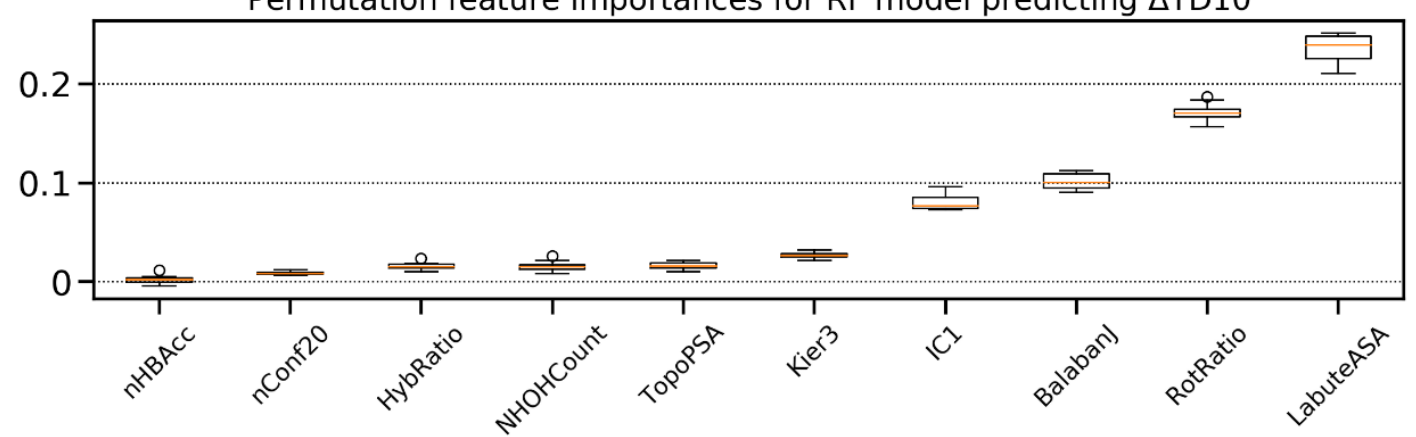

Permutation feature importances for SVC model predicting $\triangle T$ TD10

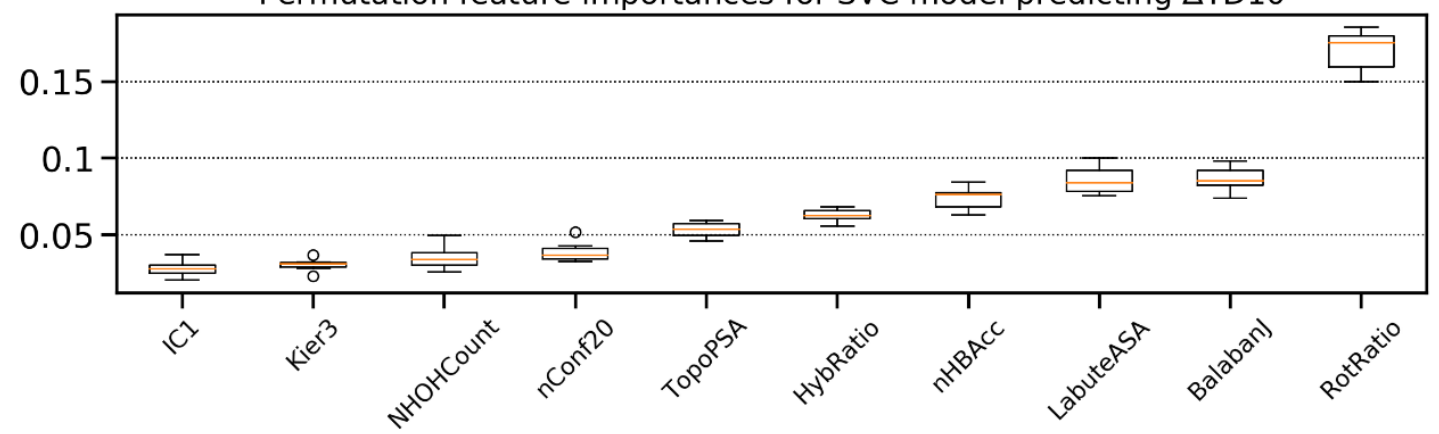

Permutation feature importances for GBC model predicting $\triangle T D 10$

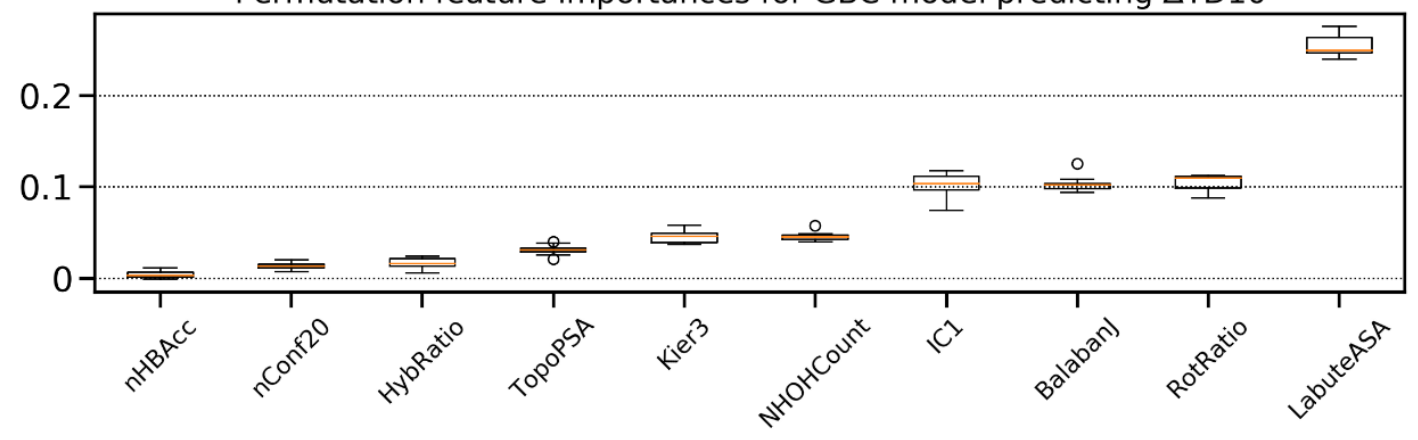


c)
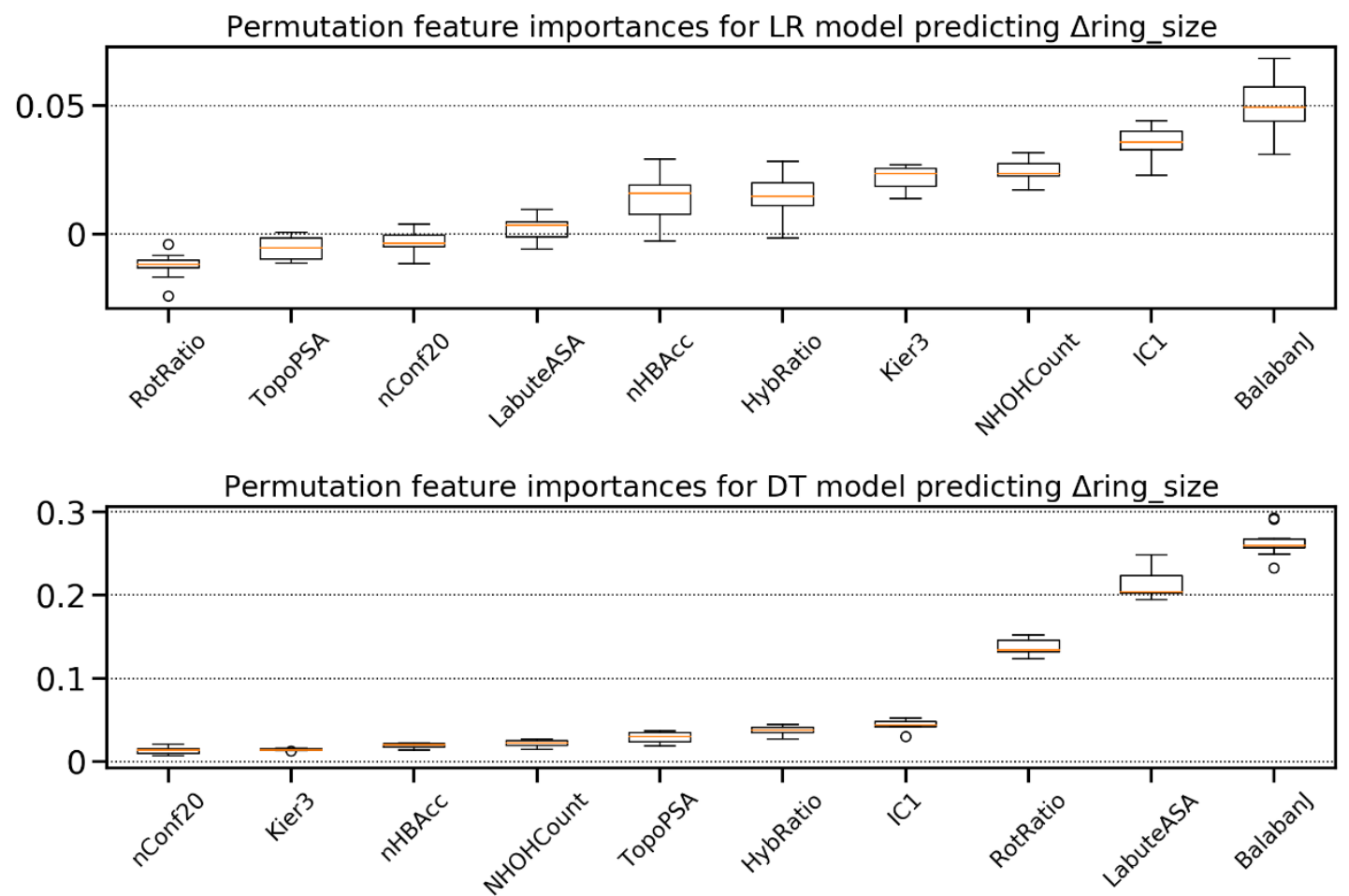

Permutation feature importances for RF model predicting $\Delta$ ring_size
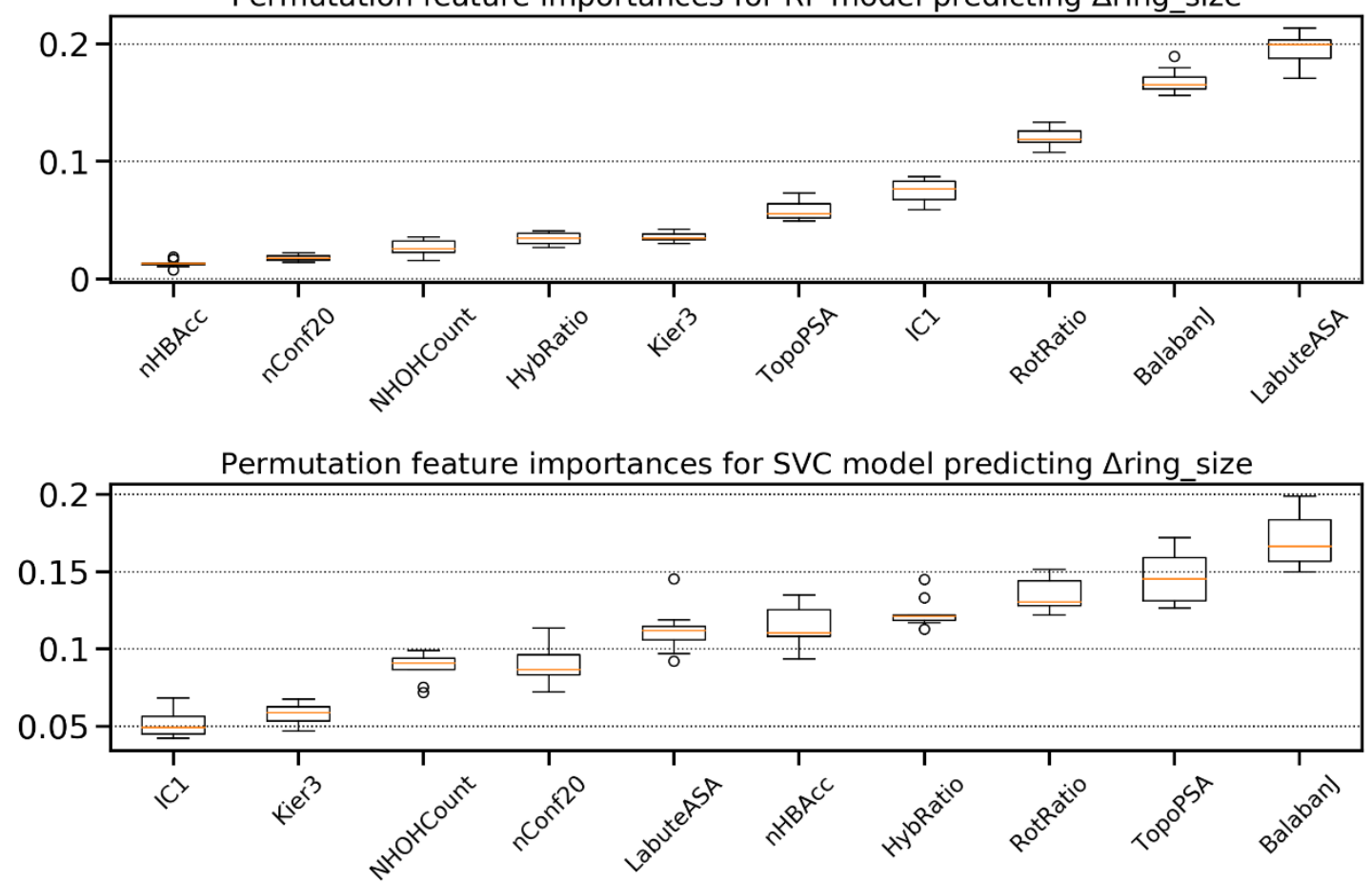

Permutation feature importances for GBC model predicting $\Delta$ ring_size

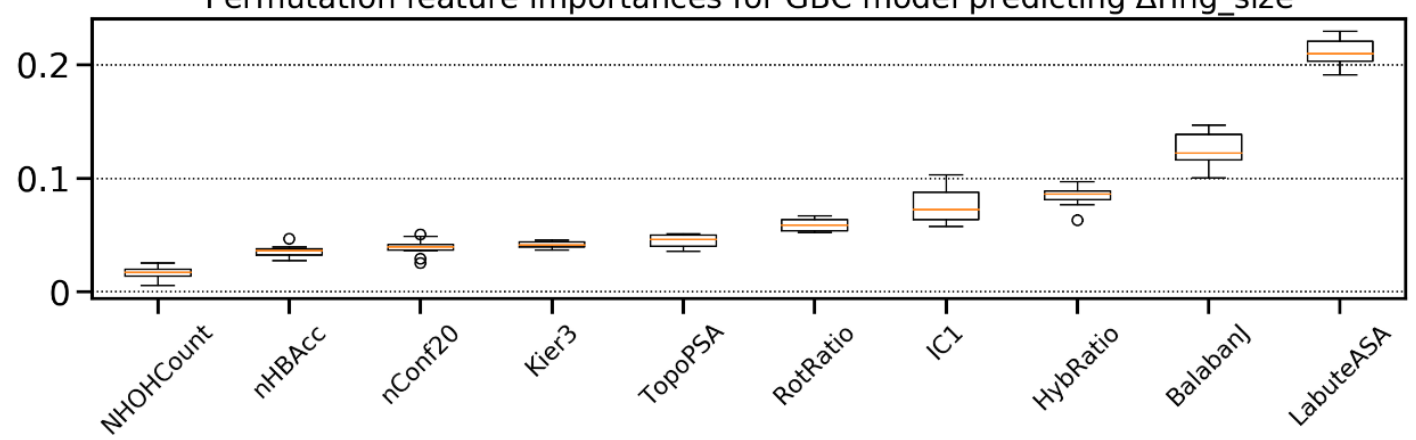


Fig. S7. The kernel density estimates for the distributions of changes in descriptors encoding extraframework ion characteristics in a set of structure pairs with (red for class 1 and green for class -1) and without (blue for class 0) changes in ring_size framework characteristic.

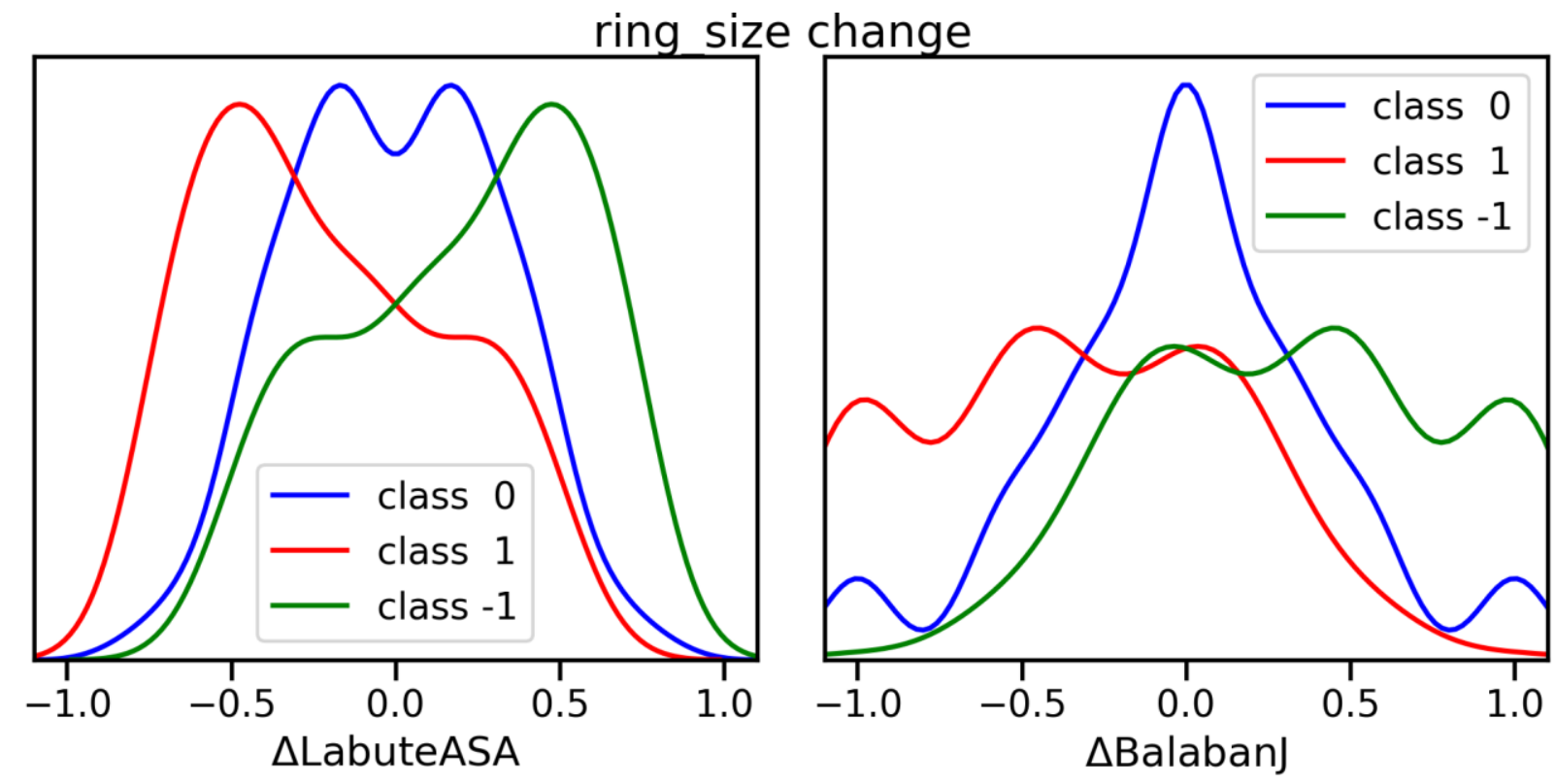


Fig. S8. Decision tree of depth three trained for the prediction of copper thiocyanate framework dimensionality (left). Scatterplots on the right show dimensionality of copper thiocyanate frameworks in crystal structures, depending on values of corresponding guest cation characteristics.
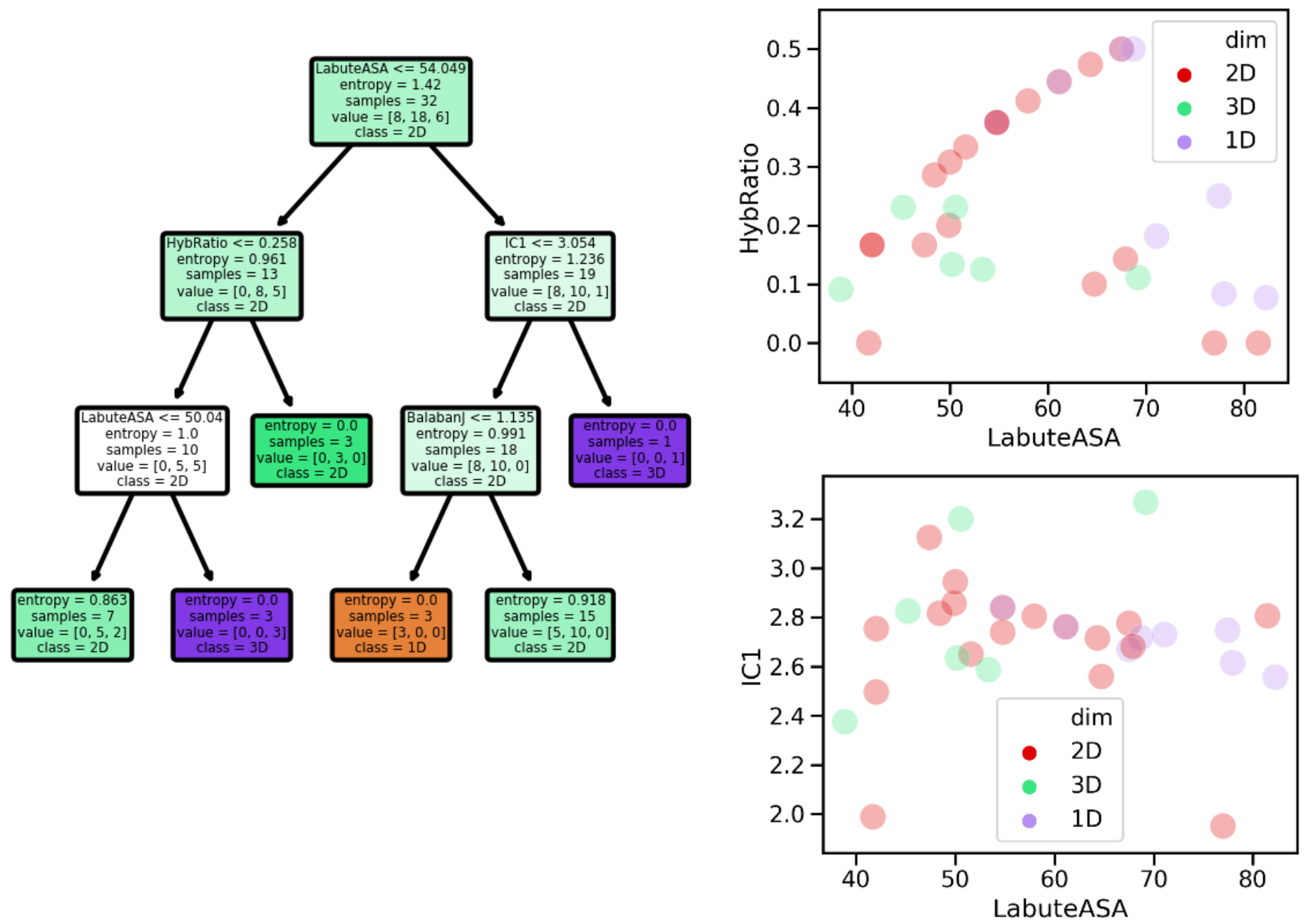